\title{
Sponge non-metastatic Group I Nme gene/ protein - structure and function is conserved from sponges to humans
}

Drago Perina', Maja Herak Bosnar ${ }^{2}$, Ružica Bago ${ }^{2}$, Andreja Mikoč ${ }^{1}$, Matija Harcet', Martina Deželjin² and Helena Ćetkovićc ${ }^{*}$

\begin{abstract}
Background: Nucleoside diphosphate kinases NDPK are evolutionarily conserved enzymes present in Bacteria, Archaea and Eukarya, with human Nme1 the most studied representative of the family and the first identified metastasis suppressor. Sponges (Porifera) are simple metazoans without tissues, closest to the common ancestor of all animals. They changed little during evolution and probably provide the best insight into the metazoan ancestor's genomic features. Recent studies show that sponges have a wide repertoire of genes many of which are involved in diseases in more complex metazoans. The original function of those genes and the way it has evolved in the animal lineage is largely unknown. Here we report new results on the metastasis suppressor gene/protein homolog from the marine sponge Suberites domuncula, NmeGp1Sd. The purpose of this study was to investigate the properties of the sponge Group I Nme gene and protein, and compare it to its human homolog in order to elucidate the evolution of the structure and function of Nme.
\end{abstract}

Results: We found that sponge genes coding for Group I Nme protein are intron-rich. Furthermore, we discovered that the sponge NmeGp1Sd protein has a similar level of kinase activity as its human homolog Nme1, does not cleave negatively supercoiled DNA and shows nonspecific DNA-binding activity. The sponge NmeGp1Sd forms a hexamer, like human Nme1, and all other eukaryotic Nme proteins. NmeGp1Sd interacts with human Nme1 in human cells and exhibits the same subcellular localization. Stable clones expressing sponge NmeGp1Sd inhibited the migratory potential of CAL 27 cells, as already reported for human Nme1, which suggests that Nme's function in migratory processes was engaged long before the composition of true tissues.

Conclusions: This study suggests that the ancestor of all animals possessed a NmeGp1 protein with properties and functions similar to evolutionarily recent versions of the protein, even before the appearance of true tissues and the origin of tumors and metastasis.

\section{Background}

The Nme family, initially called nucleoside diphosphate kinases (NDPK) or Nm23, are evolutionarily conserved proteins present in all three domains of life: Bacteria, Archaea and Eukarya [1]. Vertebrate Nme enzymes can be separated into two evolutionarily distinct groups. In humans, Group I includes Nme1-Nme4 and Group II includes Nme5-Nme9 proteins. Nme10, also known as XRP2, was the last described member and apparently

\footnotetext{
* Correspondence: cetkovic@irb.hr

'Division of Molecular Biology, Ruđer Bošković Institute, Bijenička cesta 54, 10002 Zagreb, Croatia

Full list of author information is available at the end of the article
}

has a somewhat different evolutionary history to Group I or Group II genes; it is characterized by a recent insertion of a partial NDPK domain [2]. The human Nme1 was recognized as the first metastasis suppressor and is the most studied member of the Nme family of proteins [3]. In contrast to tumor suppressor genes, metastasis suppressor genes do not abolish or diminish the tumorigenicity of a tumor, they only affect its potential to metastasise. This means that a metastasis suppressor fulfills its biological function within the processes linked to the metastatic cascade: tumor cell dissociation, invasion of the surrounding tissue, journey through the blood circulation, invasion and secondary tumor growth at

\section{Biomed Central}


a distinct site in the body [4]. In addition to melanoma [5], the metastasis suppressor activity of Nme1 has been detected in breast [6], cervical [7], hepatocellular carcinoma [8], and several other neoplastic lesions [5]. The Nme1/NDPK A and Nme2/NDPK B protein products represent two subunits of a well known, house-keeping enzyme - nucleoside-diphosphate kinase (NDPK). These subunits can assemble into the enzymatically active hexamer in all possible combinations (A6, A5B....... AB5, B6) [9]. NDPK is involved in the maintenance of the cellular NTP pool, transferring the phosphate group through the histidine phosphointermedier. Interestingly, it seems that this biochemical feature of NDPK is not responsible for its antimetastatic activity [10]. Subsequently, several other biochemical functions have been assigned to this protein: histidine kinase activity $[11,12]$, 3'-5' exonuclease and DNA cleavage activity [13], and transcriptional regulatory activity [14]. The Nme family is known to participate in numerous crucial biological events such as proliferation [15], differentiation [16,17], development $[18,19]$ and apoptosis $[20,21]$, as well as in adhesion, migration [22], and vesicular trafficking [23]. In spite of the comprehensive scientific activity in this area it is still unclear which biochemical/biological activities are responsible for Nme's antimetastatic role.

A recent evolutionary study on all known vertebrate Nmes showed that Nme1 and Nme2 arose by cisduplication [2]. Nme1 and Nme2 proteins are $88 \%$ identical in amino-acid sequences and their genes are located next to each other. Although very similar in primary structure, Nme1 and Nme2 are well distinguished by their distinct pI value: 5.8 and 8.5 , respectively. Cisduplication and the appearance of Nme1 probably occurred in a common ancestor of amniotes [2]. However, Bilitou et al. [1] observed two NmeGp1 sequences in the cnidarian Nematostella vectensis also located next to each other, and Desvignes et al. [24] found independent duplications of NmeGp1 genes in several opisthokont lineages.

Sponges (Porifera) are the simplest metazoan phylum and branched off first from the common ancestor of all animals [25,26], however, see also $[27,28]$ for an alternative view. They have changed little during evolution and can provide insight into the metazoan ancestor's genomic features [29-33]. The genome of the marine sponge Suberites domuncula encodes at least two Nme proteins. One belongs to Group I and displays a high similarity to its human Nme1 homolog (Nm23-H1), therefore it was named Nm23-SD1 when it was first described [34]. In accordance with the new nomenclature, this protein is now renamed to NmeGp1Sd. The other protein, Nme6Sd (previously Nm23-SD6) is most similar to human Group II member Nme6 protein (Nm23-H6) [34]. The total number of Nme proteins in S. domuncula is possibly greater than two because the EST database used in the $S$. domuncula Nme study [34] contained at most 4500 non-redundant cDNA sequences which is certainly far less than the total number of genes in the S. domuncula genome. Orthologues of human Nme5, Nme6 and Nme7 appeared very early in animal evolution and are present in basal metazoans - cnidarian $N$. vectensis and placozoan $T$. adhaerens as well as in choanoflagelate $M$. brevicollis genomes. Group I Nme diversified much later, after the appearance of chordates $[1,24]$. Therefore, we expect that most of the Nme enzymes in sponges are probably members of Group II.

Although the sponge $S$. domuncula NmeGp1Sd protein displays high similarity with vertebrate Nme1, the most recent Group I member, it may reflect characteristics of the protein ancestral to Group I, before the later duplications and diversifications that have occurred within this Group. Our goal was to determine the gene structure and study the biochemical characteristics and functions of the $S$. domuncula protein NmeGp1Sd. Furthermore, we wanted to test whether it can substitute for the most recent (and the most studied) Group I member human Nme1, and show a similar ability to suppress migration and, therefore, possibly even the metastatic potential of human cancer cells in vitro.

\section{Results}

Structure and evolution of sponge Group I Nme gene Group I Nme genes were analyzed in two sponges: Amphimedon queenslandica and Suberites domuncula. Only one gene coding for Group I Nme enzyme was found in the genome of the sponge A. queenslandica. A search of the corresponding EST database revealed the presence of two cDNA types with 5'untranslated regions (UTRs) $63 \mathrm{bp}$ and $76 \mathrm{bp}$ long, and therefore the existence of two transcription initiation sites. The 5'UTR region of NmeGp1Sd is 18 bp long and the transcription start site is located after oligopyrimidine tract. In $A$. queenslandica the TATA box-like motif (CCTATCAGCT) was identified in a region -33 to -24 nucleotides upstream from the transcriptional start of the shorter EST. In S. domuncula the NmeGp1Sd gene TATA box like motif (TCTAGAAATT) was found spanning from -81 to -72 nucleotides. The human Nme1 gene has no TATA box. It contains a number of motifs which may bind known transcriptional regulatory proteins such as AP-1, CTF/NF1, ACAAAG, and Ets [35]. An Ets binding sequence was found spanning from -70 to -61 nucleotides (ACCGGACGCC) in the promoter region of the NmeGp1 gene from sponge A. queenslandica and from -365 to -374 nucleotides (ACAGGATATA) in the promoter region of the NmeGp1Sd gene from sponge $S$. domuncula. Chen et al. 
[36] showed that the presence of a motif typical for AP-1 transcriptional elements in the 5' region of the human Nme1 gene is essential for promoter activity. A putative site for AP-1 in the A. queenslandica NmeGp1 5 ' flanking region was found spanning from -117 to -125 nucleotides (TTGACTCAG) and in S. domuncula NmeGp1 from -130 to -138 nucleotides (ATGTCTCAG). The structure of sponge NmeGp1 promoter regions is shown in Additional file 1.

The Nme gene from A. queenslandica is 654 bp long from the ATG start codon to the TAG stop codon. It has four short introns with lengths of 47, 47, 57 and 47 bp, respectively. The NmeGp1Sd gene from S. domuncula coding region and introns encompasses $736 \mathrm{bp}$ from the ATG start codon to the TAA stop codon and contains three introns $(89,57$ and $134 \mathrm{bp})$. Their positions and phases are shown in Figure 1. Multiple sequence alignment of NmeGp1Sd gene homologs was produced and used to compare intron positions. The results are shown in Figure 1. NmeGp1Sd gene lacks the fourth intron which is present in the homolog from $A$. queenslandica.

As mentioned before, two NmeGp1 genes in the cnidarian $N$. vectensis were found. Like Nme1 and Nme 2 in mammals and lizard, these two genes are located next to each other. Therefore, we checked neighboring genes in the genome of $A$. queenslandica and found that the NmeGp1 gene is surrounded by unc50 and acad 8 genes and not by another Nme homolog. Extensive searches of the A. queenslandica genome [32] and S. domuncula

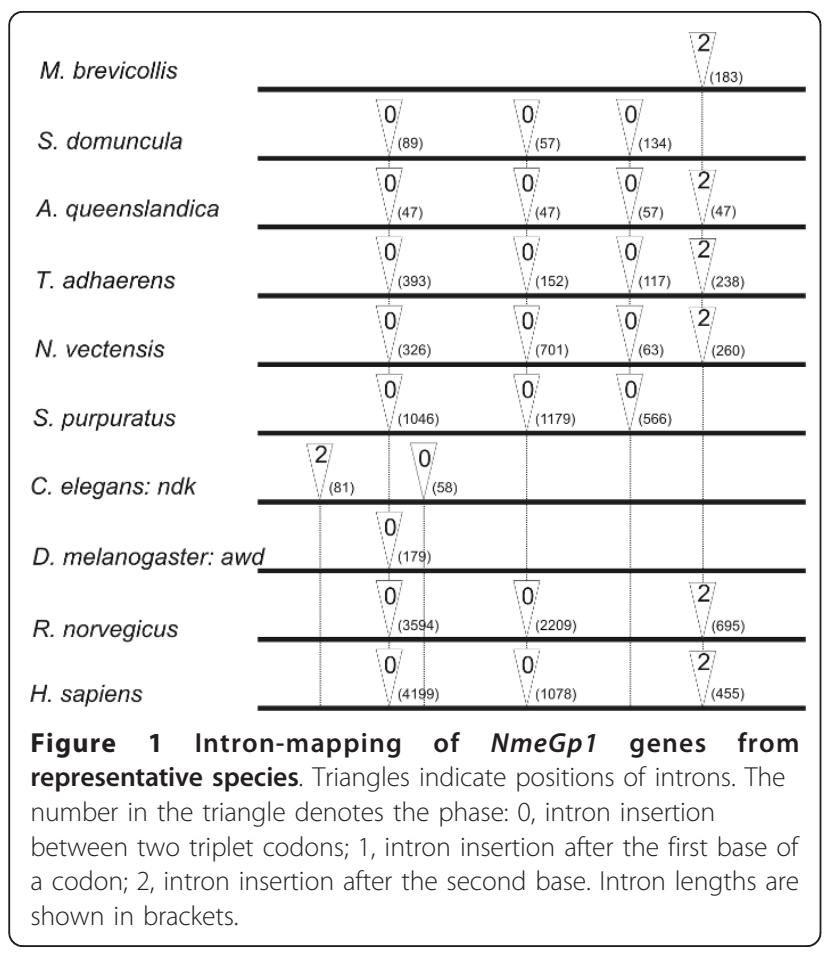

cDNAs [33] revealed only one NmeGp1 gene in each of these two demosponges. Independent duplications have also been identified in Branchiostoma floridae and Ciona intestinalis [2] as well as more recently in other opisthokonts [24]. Searches of NCBI's dbEST revealed the presence of cDNAs that encode two NmeGp1 proteins in the calcareous sponges Sycon raphanus [GenBank:AM764253 and GenBank:AM764213] and Leucetta chagosensis [GenBank:GO094744 and GenBank:GO094741]. Phylogenetic analysis shows that distinct sponge proteins from the same species clearly group together, indicative of independent duplications (Additional file 2). The cnidarian Hydra magnipapillata also has at least two Group I member genes which are not located next to each other in the genome. Demosponge proteins cluster in a poorly supported and poorly resolved branch together with cnidarian, placozoan, S. purpuratus and B. floridae homologs while calcarean proteins form a separate branch.

\section{The sponge NmeGp1Sd protein forms a hexamer}

Various oligomeric structures such as hexamers, tetramers, dimers, and monomers were found after crosslinking the NmeGp1Sd protein with glutaraldehyde. Recombinant NmeGp1Sd appears to be predominantly in the hexameric form (Figure 2A). A similar oligomeric pattern was observed with the recombinant human Nme1 protein. The hexameric form was expected due to the conservation of the KPN loop (residues 91 to 113 in NmeGp1Sd). Each KPN loop interacts with two others with helices $\alpha 1$ and $\alpha 3$, and with the four terminal residues [1], well preserved in the sponge NmeGp1Sd (residues 148 to 151). Residues Lys 30, Pro 95 [37] and Ser 119 [38] known to be crucial for
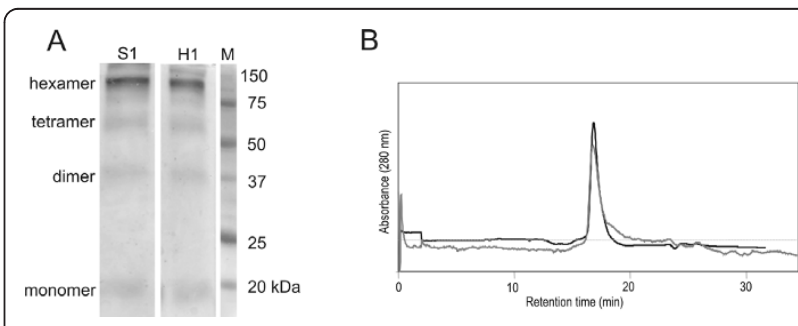

Figure 2 Oligomerization of sponge NmeGp1Sd protein and human Nme1 as control. (A) Cross-linking NmeGp1Sd (S1) and Nme1 (H1) with glutaraldehyde. Purified recombinant protein NmeGp1Sd $(8 \mu \mathrm{g})$ was pre-incubated in PBS at room temperature with $25 \mathrm{mM}$ of glutaraldehyde to initiate the cross-linking. After quenching, the reaction product was subjected to $12.5 \%$ SDS-PAGE gel followed by staining with Coomassie brilliant blue. (B) Size exclusion chromatography. Recombinant human Nme1 and sponge NmeGp1Sd were loaded onto Bio-Sil SEC 250 gel-filtration columns ( $300 \mathrm{~mm} \times 7.8 \mathrm{~mm}$ ) and eluted with $\mathrm{Nm} 23$ buffer at a flow rate of $0.5 \mathrm{~mL} / \mathrm{min}$. Black line represents human $\mathrm{Nme} 1$ and grey line sponge NmeGp1Sd protein. 
hexamer formation are also conserved in the sponge NmeGp1Sd (Additional file 3). Thus, we can conclude that all the residues necessary for the hexameric structure are present in the sponge NmeGp1Sd enzyme, and known residues that could impede this structure are absent. The hexameric structure of sponge NmeGp1Sd was also confirmed by gel filtration (Figure 2B). Previously described human Nme1 [39] was used as marker. Overlapping gel filtration peaks confirm our finding that both proteins have a similar molecular weight as well as hexameric structure.

The sponge NmeGp1Sd protein has a similar level of kinase activity as the human Nme1 but shows nonspecific DNA-binding activity

The specific kinase activities of the sponge NmeGp1Sd and human Nme1 enzymes were measured in parallel and found to be $496 \mathrm{U} / \mathrm{mg}$ and $407 \mathrm{U} / \mathrm{mg}$, respectively. In comparison, Ma et al. [13] found the kinase activity of Nme1 protein to be $570 \mathrm{U} / \mathrm{mg}$ while Bago et al. [39] observed $237 \mathrm{U} / \mathrm{mg}$. The specific activities of the sponge NmeGp1Sd enzyme and human Nme1 kinase were similar and within the previously reported ranges for the human protein.

Purified NmeGp1Sd protein is able to bind nonspecifically to single-stranded circular DNA (sscDNA) (Figure 3). Binding activity was observed with concentration of $0.8 \mu \mathrm{M}$, manifested by increase in DNA band retardation. The majority of the protein-DNA complexes at concentrations of NmeGp1Sd $>1.7 \mu \mathrm{M}$ exist in the form of big aggregates which stack in the wells (Figure 3, left). Human Nme1 did not display an ability to bind sscDNA, while human Nme2 binds sscDNA and shows the same DNA band retardation effect as sponge NmeGp1Sd. However, higher concentration $(>4 \mu \mathrm{M})$ of

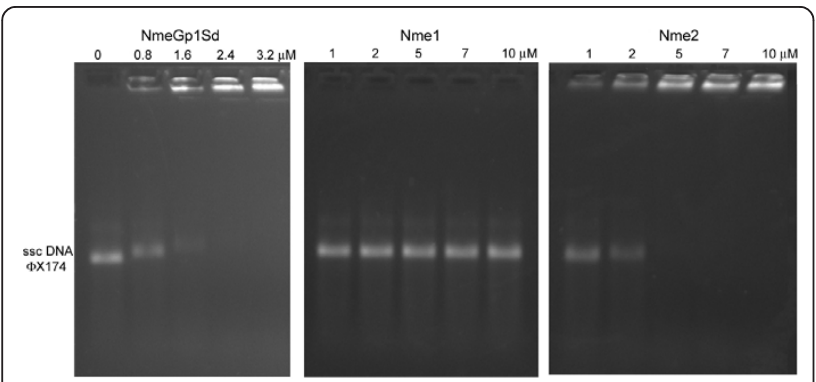

Figure 3 DNA-binding activity of the NmeGp1Sd protein. Human Nme1 and Nme2 proteins were used as control. The reaction was performed in buffer containing $40 \mathrm{mM}$ Tris-acetate (pH 7.5) and $12 \mathrm{mM} \mathrm{MgCl}_{2}, 30 \mathrm{nM}$ of single-stranded circular (ssc) DNA from bacteriophage $\varphi$ X174 and purified NmeGp1Sd protein as indicated. $40 \mathrm{mM}$ Tris-acetate (pH 7.5) containing 50\% glycerol and $0.01 \%$ bromophenol blue was added before the products were analyzed in $0.6 \%$ agarose gel. human Nme2 protein was needed to form the same amount of aggregates (Figure 3, right).

\section{The sponge NmeGp1Sd does not cleave negatively supercoiled DNA}

Nme2 is involved in DNA structural changes necessary for the activity of the $c-m y c$ promoter. It binds to the NHE sequence of the $c-m y c$ promoter cloned into pUC19 which yields mostly open circle (nicked circular) plasmid [40]. We have considered the possibility that the sponge NmeGp1Sd may already have DNA topoisomerase-like activity similar to the human Nme2. To test this activity, NmeGp1Sd protein was incubated with supercoiled pUC19 plasmid containing the 57-bp c-myc NHE sequence. We used topoisomerase I, Nme1 and Nme2 proteins as controls. Control topoisomerase I and human Nme2 cleaved negatively supercoiled plasmid DNA unlike NmeGp1Sd and human Nme1 which did not display this activity (Figure 4).

\section{The human cell recognizes the sponge Nme as its own}

The objective of testing the localization and function of sponge NmeGp1Sd in well established human in vitro systems was to reveal whether the sponge NmeGp1Sd can be recognized as the domestic protein in the human cell and whether it can replace its human homolog. To compare the subcellular localization of human Nme1 and NmeGp1Sd in HEp-2 cells we transiently transfected the cells with pEGFPN1-Nme1 and pDsRedN1NmeGp1Sd. We analyzed the cells 48 hours post transfection using confocal laser scanning microscopy (Figure 5). The localization of Nme1-GFP was mainly the same as in a previous study [41] in which Nme1 was fused to the C-terminus of GFP (reverse orientation). The signal is mainly present in the cytoplasm, has a punctiform structure with occasional bigger highly

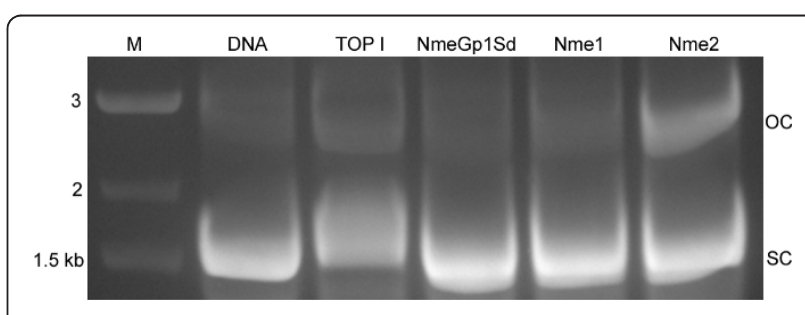

Figure 4 Cleavage of negatively supercoiled pUC19-NHE plasmid DNA. Reaction was assembled in $50 \mathrm{mM} \mathrm{KCl}, 10 \mathrm{mM}$ $\mathrm{MgCl}_{2}, 50 \mathrm{mM}$ Tris $\mathrm{HCl} \mathrm{pH} 7.5,0.5 \mathrm{mM}$ DTT, $30 \mu \mathrm{g} / \mathrm{mL}$ BSA with $500 \mathrm{ng}$ of pUC19-NHE plasmid DNA (lane DNA) and $500 \mathrm{ng}$ of proteins topoisomerase I, NmeGp1Sd, human Nme1 and Nme2, as indicated. After 1\% SDS, $10 \mathrm{mM}$ EDTA and proteinase $\mathrm{K}$ treatment $(200 \mu \mathrm{g} / \mathrm{mL})$ samples were analyzed in 1\% agarose gel in TAE buffer and then stained with $0.5 \mu \mathrm{g} / \mathrm{mL}$ ethidium bromide for $30 \mathrm{~min}$. SC marks super coiled DNA, while OC marks open circle/nicked circular DNA. 


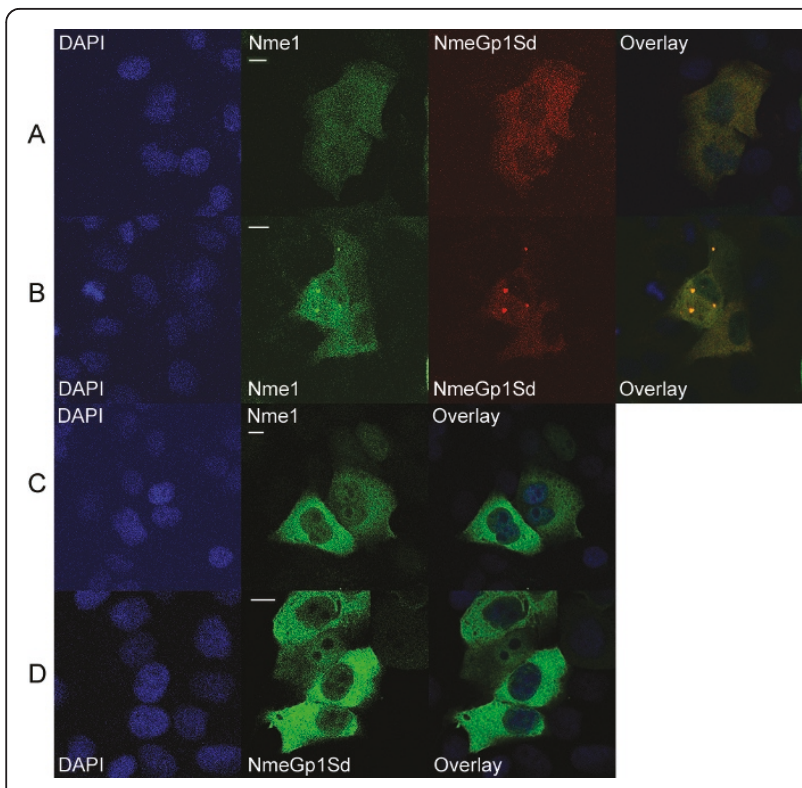

Figure 5 Subcellular localization of NmeGp1Sd and human Nme1. A) HEp-2 cells transiently transfected with pEGFPN1-Nme1 (green fluorescence) and pDsRed-NmeGp1Sd (red fluorescence). The signal is visible mainly in the cytoplasm. The overlay (yellow) shows colocalization of the human and sponge homologs. B) HEp-2 cells transiently transfected with pEGFPN1-Nme1 (green fluorescence) and pDsRed-NmeGp1Sd (red fluorescence). The signal is visible mainly in the cytoplasm. The "granum-like" structures are visible in the cytoplasm, and they are formed with both, the human and the sponge homolog. The overlay (yellow) displays the subcellular colocalization of human and sponge homologs. C) HEp-2 cells transiently transfected with pEGFPN1-Nme1. The signal is present in the cytoplasm, but also in the nucleus, although not so prominent. D) HEp-2 cells transiently transfected with pEGFPN1-NmeGp1Sd. The signal is present in the cytoplasm and in the nucleus. Bar $=10 \mu \mathrm{m}$.

fluorescent "granum-like" structures present in the cytoplasm, often, but not exclusively, located adjacent to the nucleus. Nme1 can also very clearly be seen in a portion of the nuclei. The NmeGp1Sd-DsRed reveals the same localization pattern which could be seen in separate figures and merged with human Nme1 (yellow staining). To confirm the NmeGp1Sd nuclear localization we performed additional experiments and transfected HEp-2 cells with pEGFPN1-NmeGp1Sd since the GFP has a brighter fluorescence than the DsRed and is, therefore, easier to analyze. These experiments confirmed the occasional presence of NmeGp1Sd in the nucleus. The fluorescent NmeGp1Sd also seems to form "granumlike" structures and they colocalize with the ones formed by human Nme1. These experiments show that the human and the sponge Nme colocalize - adopt the same distribution in all transfected HEp-2 cells analyzed, at any time-point. These experiments encouraged us to further analyse the interaction of human Nme1 and NmeGp1Sd in human cells.

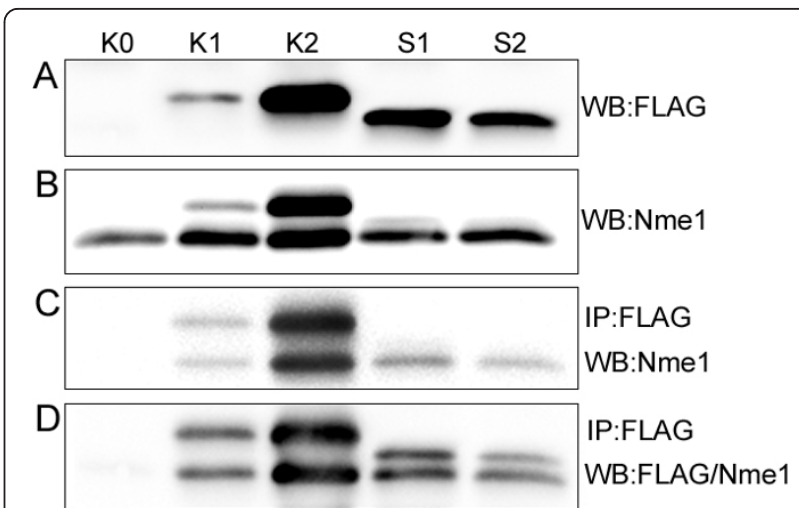

Figure $6 \mathrm{Nme1/NmeGp1Sd} \mathrm{complex} \mathrm{formation} \mathrm{analysis:}$ A) Input control: Cell lysates from control KO (CAL 27 cells stably transfected with pCDNA3 vector), $\mathrm{K} 1$ and $\mathrm{K} 2$ (CAL 27 cells stably transfected with pcDNA3FLAG/Nme1 construct) and S1 and S2 (CAL 27 cells stably transfected with pcDNA3FLAG/NmeGp1Sd constructs) tested with anti-FLAG antibody. B) Cell lysates from control KO (CAL 27 cells stably transfected with pcDNA3 vector), K1 and K2 (CAL 27 cells stably transfected with pcDNA3FLAG/Nme1 constructs) and S1 and S2 (CAL 27 cells stably transfected with pcDNA3FLAG/ NmeGp1Sd constructs) tested with anti-Nme1 antibody. C) Immunoprecipitation: FLAG/Nme1 (K1 and K2) and FLAG/ NmeGp1Sd (S1 and S2) were immunoprecipitated with anti-FLAG M2 affinity gel and immunoblotted with anti-Nme1 antibody. KOcontrol (clone with "empty" construct). FLAG/Nme1 produces heteromers with exogenous (upper band) and endogenous (lower band) Nme1. FLAG/NmeGp1Sd produces complexes with the endogenous (human) Nme1 while the upper band (exogenous, FLAG/NmeGp1Sd) cannot be seen, since the antibody is specific for human Nme1. D) Immunoprecipitation: FLAG/Nme1 (K1 and K2) and FLAG/NmeGp1Sd (S1 and S2) were immunoprecipitated with anti-FLAG M2 affinity gel and immunoblotted with anti-FLAG and anti-Nme1 antibody. KO-control (clone with "empty" construct). FLAG/Nme1 produces heteromers with exogenous (upper band) and endogenous (lower band) Nme1. FLAG/NmeGp1Sd produces complexes with the endogenous (human) Nme1. The upper band (exogenous, FLAG/NmeGp1Sd) is visible, since it is stained with antiFLAG antibody.

In Western blot analysis a few CAL 27 clones expressing NmeGp1Sd were identified, and two of them (S1 and S2) were chosen for further analysis (Figure 6A and $6 \mathrm{~B})$. To test the possible interactions of sponge and human Nme proteins we used the previously produced Nme1 overexpressing CAL 27 clones K1 and K2 [39]. To test if sponge FLAG/NmeGp1Sd forms complexes with endogenous human Nme1, FLAG was immunoprecipitated from cell lysates of FLAG/Nme1 overexpressing clones (K1 and K2), and FLAG/NmeGp1Sd expressing clones (S1 and S2) with anti-FLAG M2 affinity gel. The results of the Western blot analysis with anti-Nme1 or anti-Nme1 and anti-FLAG antibody reveal that FLAG/NmeGp1Sd forms complexes with the endogenous Nme1 (lower band in Figure 6C and 6D). Therefore, we have proved that the human Nme1 recognizes the sponge variant as a partner. The final confirmation 


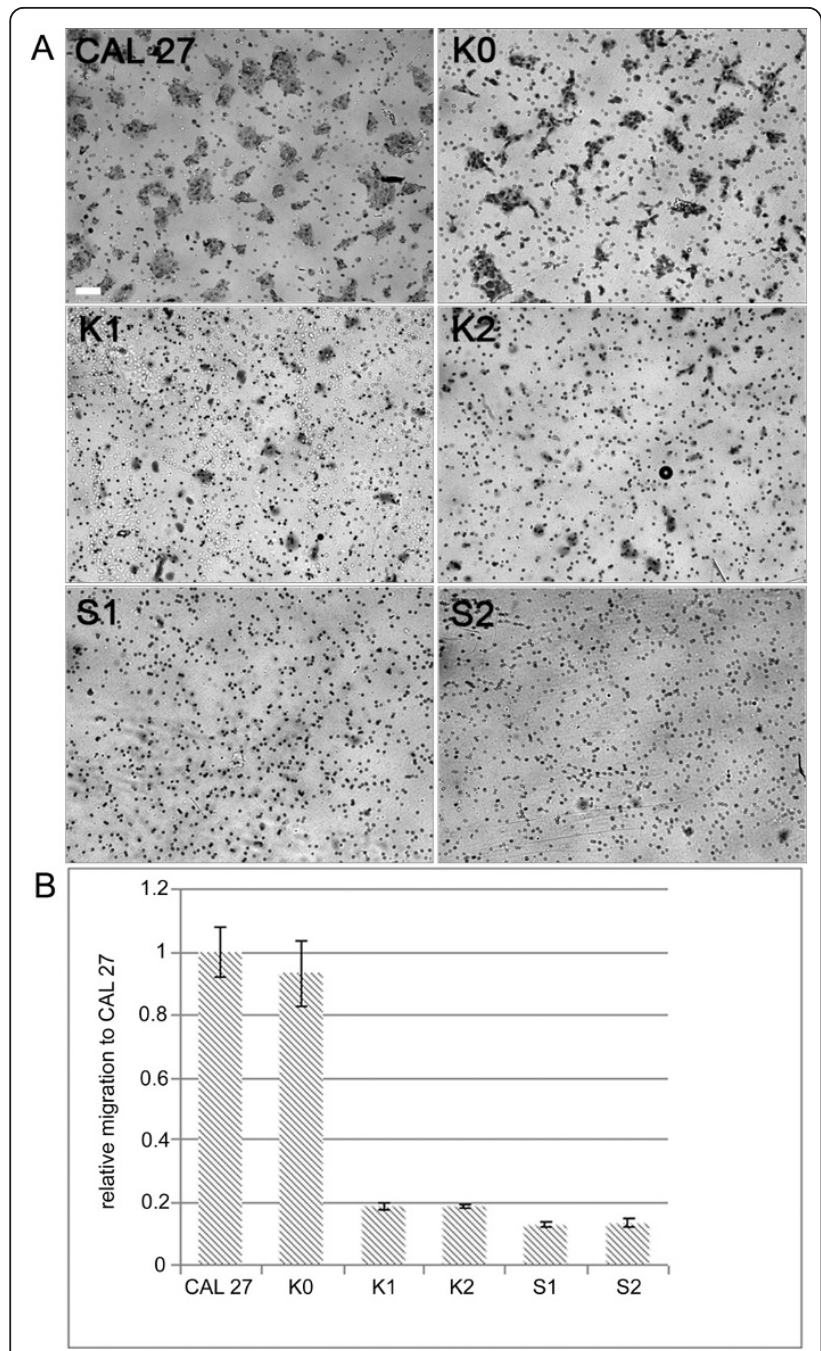

Figure 7 Migration assay. A) CAL 27 cells, stable clones transfected with pcDNA3 vector (K0), pcDNA3FLAG/Nme1 ( $\mathrm{K} 1$ and $\mathrm{K} 2$ ) and pcDNA3FLAG/NmeGp1Sd (S1 and S2) were tested for migration potential in Boyden chambers. The cells were stained with crystal violet. The images were recorded by inverted light microscope. The $\mathrm{K}$ and $\mathrm{S}$ clones clearly show lower migratory potential compared to untransfected CAL 27 and $\mathrm{KO}$ control cells. Bar $=50 \mu \mathrm{m}$. B) The results are presented as a relative number of migrated cells ( \pm SD) compared to CAL 27. The results were produced by ImageJ program measuring the membrane area covered with migrated cells. The results show that $\mathrm{S} 1$ and $\mathrm{S} 2$ clones as well as $\mathrm{K} 1$ and $\mathrm{K} 2$ exhibit diminished migration potential compared to CAL 27 and CAL 27 transfected with "empty vector" (KO).

of our theory that the human cell recognizes the sponge protein as its own comes from tests in Boyden chambers. The intention of this experiment was to test the migration potential of CAL 27 cells stably expressing sponge NmeGp1Sd, and compare it with the human Nme1 overexpressing cells that show diminished migratory potential compared to control, CAL 27 cells. Figure 7 shows that NmeGp1Sd expressing clones, (as well as human Nme1 overexpressing clones as previously published [39]) have substantially diminished migratory potential. Therefore, NmeGp1Sd suppresses motility of CAL 27 cells. The same result was observed in three independent experiments.

\section{Discussion}

The objective of this study was primarily to determine the structure and biochemical characteristics of the NmeGp1Sd in order to gain a deeper insight into the evolution of metazoan Nme proteins and their functions.

Sponge Group I Nme genes are intron-rich and these introns are relatively short. The same has been found for introns in several other sponge genes $[42,43]$ and recently in $A$. queenslandica genome where median intron size is $80 \mathrm{bp}$ [32]. The fourth intron (Figure 1) is likely the most ancient because it is also found in a choanoflagellate Group I Nme homolog. We conclude that the ancestral metazoan Group I Nme gene was intron-rich and probably had all four introns that are still present in most extant basal metazoan homologs. The ancestral gene structure is also well preserved in vertebrate homologs with three out of four introns present. D. melanogaster has only one of the ancestral introns and C. elegans lost all ancestral introns and gained two new ones which likely reflect accelerated evolution in these lineages. Analysis of sponge NmeGp1 promoters showed that some of the motifs essential for human promoter activity are also present in sponges. We did not find these motifs in the corresponding choanoflagellate promoter under the same search parameters, which indicates a possible change in Nme1 regulation in the metazoan lineage.

The phylogenetic tree (Additional file 2) is generally not well resolved or supported, as in comparable earlier studies $[1,2,24]$. Nonetheless, it does provide some new evidence on Group I Nme evolution. Group I Nme gene duplicates in $N$. vectensis had been proposed to indicate that the split into Nme1 and Nme2 might have occurred very early in metazoan evolution [1]. Another recent study [24] showed that duplications of Group I Nme genes occurred independently more than once in invertebrates, besides well described duplications and diversifications of Group I Nme to Nme1, 2, 3 and 4 in vertebrate lineage, and that duplication in $N$. vectensis is also an independent event unrelated to the origin of Nme1 and Nme2. Our results confirm these findings and additionally demonstrate independent duplications in basal metazoans, even within the same lineages (cnidarians and calcareous sponges).

The metastasis suppressor genes Nme are highly conserved in opisthokonts. This implies that they have important roles in basic cellular functions. A number of 
studies revealed several potential biological roles of Nme genes/proteins, but most are not confirmed in vivo. The expression of the $D$. discoideum Nme homologues gip17 and guk7.2 is modulated during the developmental phases of cell growth or aggregation due to starvation [44]. In Drosophila, Nme/Awd is required for the proper differentiation of many tissues including the brain, eye, and female reproductive system [45]. Nme protein accumulation is coincident with the functional differentiation of multiple epithelial tissues in the developing mouse [18]. These and many other studies demonstrate that Nme proteins have a critical role in differentiation and development - processes that involve cell migration, a prerequisite for metastasis formation [1].

The sponge protein NmeGp1Sd was compared primarily with human Nme1 (and Nme2) for several reasons: i) The NmeGp1Sd is highly similar to vertebrate Nme1/Nme2 in primary structure, ii) human Nme1/ Nme2 proteins are the most studied and well characterized Group I Nme members iii) Nme1 is linked to metastasis suppression, but the suppression mechanism is not well understood. We expected that NmeGp1Sd analysis and the discovery of its biochemical features would shed some more light into the mechanisms connecting various Nme1 functions and its antimetastatic activity. A recent study by Domazet-Lošo and Tautz [46] shows that, contrary to what might be intuitively expected, only a subset of cancer genes appeared simultaneously with multicellularity in animals and that the new genetic processes and gene functions that emerged at the basis of the metazoan lineage are major innovations which enabled complex interactions between metazoan cells. Demosponges have only one Group I Nme protein (whereas amniotes have four with potentially different functions), which is probably the ancestral condition for metazoans.

Our results show that the sponge NmeGp1Sd possesses some biochemical characteristics typical for human Nme1 and some typical for human Nme2 protein. Recombinant NmeGp1Sd appears to be predominantly in the hexameric form like human Nme1 protein which was confirmed by gel filtration (Figure 2A and 2B). The sponge NmeGp1Sd and human Nme1 enzymes also have similar levels of kinase activity. Human Nme1 did not display an ability to bind sscDNA, however, human Nme2 can bind sscDNA and showed the same DNA band retardation effect as the sponge homolog. Accordingly we decided to check the possibility that the sponge NmeGp1Sd is functionally more similar to Nme2 than to Nme1. Unlike Nme1, Nme2 is able to cleave $c-m y c$ NHE sequence [40]. Therefore, we tested whether the sponge NmeGp1Sd also has DNA topoisomerase-like activity and found out that it is not able to cleave $c$-myc NHE sequence. We hypothesize that this was the ancestral condition of the Nme Group I protein before duplications and functional diversifications within the Group I Nme. Sponge NmeGp1 may have retained the multiple functions, while these functions have been partitioned between different vertebrate Group I Nme proteins. Recent studies showed that sponge genomes are comparable to genomes of "higher" complex animals, including vertebrates, in terms of gene number and functional repertoire [32,33]. It was suggested that complex animals differ from their early simple relatives mainly in the more complex regulation of similar sets of genes. From that point of view, it could be speculated that the ability of Nme2 to cleave $c-m y c$ is a relatively recent addition to the regulatory networks.

Based on this evidence we then asked the following: What is the biological function of NmeGp1Sd in its native environment - the sponge cell? As it can suppress the migration of CAL 27 cells e.g. replaces the human Nme1 it could thus be involved in migration and/or, possibly, adhesion of sponge cells. It has previously been found that sponges possess genes/proteins for many advanced physiological processes usually linked to more complex metazoan phyla [31,47]. Migration of cells in complex "higher" animals is limited to specific processes such as embryonic development and immune response and is closely controlled. Cells are anchored in the developed tissues they belong to and do not migrate if not stimulated (with the exception of cells undergoing malignant transformation). The anchorage and migration of cells depends on a vast number of molecules such as cadherines, integrins, fibronectin and collagen. Although sponges do not possess true tissues and organs, they do possess simple varieties of the mentioned proteins $[48,49]$. Furthermore, they have complex pelagobenthic life cycles which include development to a larval phase and metamorphosis to sedentary adult form [50]. Cell migration is present in sponges in three different processes: regeneration, larval development and the movement of amoeboid cells through the adult mesohyl; the space between the external and internal cell layers which is composed of galectin, collagen, fibronectin-like molecules and dermatoponin [51]. Although the mesohyl is not a homogenous, organized structure, it resembles a primitive extracellular matrix (ECM). Therefore, both the movement of amoeboid cells through the mesohyl in sponges and the movement of cells through ECM in vertebrates may originate from the same ancient precursor process present in the metazoan last common ancestor. From an evolutionary point of view, tumors likely developed together with the evolution of tissues and organs. It is not yet defined which biochemical functions of Nme are crucial for its metastasis suppressor activity. It is, however, to be expected that its main biological function (besides the 
well defined NDPK function), would not be to suppress metastasis in "higher" Metazoa but to control normal physiological processes (adhesion, migration) which, if left without control, could lead to metastasis formation. As sponges do not possess tissues and organs they are probably also incapable of forming tumors. The demonstration of the fact that the sponge NmeGp1Sd can replace human Nme1 in human tumor cells encourages us to propose that the NmeGp1 (at least Nme1) function responsible for regulating adhesion/migration of cells was established in the metazoan ancestor well before the Cambrian explosion i.e. before the appearance of diverse groups of multicellular Metazoa. Since tumors likely started developing in parallel with the development of true tissues and organs, this ancient function of Nme1 was then adapted to act in a new highly significant role - the suppression of metastasis.

\section{Conclusions}

In conclusion, our study emphasizes three major points: i) the single sponge Group I Nme gene and Nme protein of Suberites domuncula probably reflects the characteristics of the ancestral Group I Nme gene/protein, that existed prior to the duplication of this group in vertebrates and other lineages; ii) NmeGp1Sd possesses many biochemical properties of the most recent Group I variant, the Nme1 human homolog; iii) NmeGp1Sd interacts with human Nme1 and can replace it in some biological functions which are usually associated with "higher" metazoans, specifically in metastasis suppression. Therefore, we presume that the Group I Nme gene/protein of the metazoan last common ancestor was structurally and functionally similar to the multifunctional enzyme it is today.

\section{Methods}

\section{Sequence and phylogenetic analyses}

For genomic DNA preparation, the specimens of $S$. domuncula were cut into pieces, frozen in liquid nitrogen, ground to fine powder from which total DNA was isolated using the Genomic DNA Purification kit (QIAGEN). Sponge NmeGp1Sd gene was amplified by PCR, using KOD XL DNA polymerase (Novagen) and primers specific for the 5'-(GCTTTTCTGTGTGT GGAGCTT) and 3'-(TCCCTAAACCAAAAAGTTACTCAT) ends of the cDNA coding sequence [GenBank: AY764256]. The amplified fragment was separated on a $0.8 \%$ agarose gel, purified and sequenced. The reverse specific primer 5'- CATGGCAACCATCTTGAAGCC -3 ' was used in conjunction with the vector specific primer T3 to amplify the 5' upstream region from translation start of the nmeGp1Sd gene on the genomic library from S. domuncula [52].
Nucleotide sequences were stored and analyzed using Lasergene (DNAStar, Madison, WI). Multiple sequence alignments (MSA) were performed with CLUSTALX [53]. The exact position and the phase of each intron were verified by manual inspection. MEGA4 [54] was used to construct and bootstrap phylogenetic trees.

The homolog of the NmeGp1Sd gene from the sponge Amphimedon queenslandica was identified in the genome (contig13509) available at http://spongezome.metazome.net/cgi-bin/gbrowse/amphimedon/ [32]. Homology searches and sequence retrievals were done using BLAST (NCBI, NIH, Bethesda, MD, USA: http://www. ncbi.nlm.nih.gov). NmeGp1 sequences from sponges Leucetta and Sycon were identified by blastn with $n m e G p 1 S d$ sequence against NCBI's dbEST limited to Porifera. Hamming clustering method for TATA signal prediction in eukaryotic genes was used for identifying TATA-box http://www.itb.cnr.it/sun/webgene/ [55]. TFSEARCH http://www.cbrc.jp/research/db/TFSEARCH. html and TRANSFAC database [56], with a default threshold score of 85 were used for searching -400 bp 5' flanking region for possible transcription factors binding sites.

\section{Plasmid constructions}

We used the same set of primers as for NmeGp1Sd gene to perform PCR reactions on the $S$. domuncula cDNA library. The PCR generated cDNA fragment was cloned into pBluescript vector (Stratagene). The cDNA for NmeGp1Sd was recloned into pET15b (Novagen) using PCR into BamHI (5'-TTCCCGGATCCAAAAAGT TACTCATAGATC-3') and NdeI (5'-GCTTTATACA TATGACAACCGAGCGTACC-3') restrictions sites of pET15b expression vector downstream from the thrombin cleavage site. For colocalization assay, the full-length coding sequence NmeGp1Sd was cloned in frame into pEGFPN1 and pDsRedN1 expression vectors (Clontech, USA). The fragment of 541 bp was amplified using 5'GCTTTCTCGAGATGACAACCGAGCGTACC-3' and 5'-GTAGACGGATCCTTCTCATAGATCCATTGCTG3' primers. The PCR product was digested with XhoI and $B a m \mathrm{HI}$, and ligated with the XhoI/BamHI sites of pEGFP-N1 and pDsRedN1 expression vectors. The pEGFPN1-Nme1 was a kind donation of Dr. Marie-Lise Lacombe, Faculty of Medicine St. Antoine, Paris, France. NmeGp1Sd cDNA was amplified using a forward primer containing the FLAG sequence (F: 5'-GTCTAGGGATC CACGAGATGGACTACAAGGACGACGACGATAA GATGACAACCGAGCGT-3') and the reverse primer (R: 5'-CTAGACGAATTCTTATTACTCATAGATC CATTGCTGTTCTGTGGG-3'). The PCR product was cloned into the $B a m \mathrm{HI} / E c o$ RI sites of eukaryotic expression vector pcDNA3 (Invitrogen). 


\section{Protein expression and purification}

NmeGp1Sd was overproduced in Escherichia coli strain BL21 tagged with six histidine residues at the $\mathrm{N}$ terminus and purified to homogeneity from bacterial lysates using nickel affinity chromatography. E. coli strain BL21 harboring the plasmid construct was grown to 0.6 at $\mathrm{OD}_{600}$ and induced with $0.8 \mathrm{mM}$ IPTG for 3 hours at $37^{\circ} \mathrm{C}$. Cells were incubated $30 \mathrm{~min}$ on ice in lysis buffer $(50 \mathrm{mM}$ Tris $\mathrm{HCl}, 300 \mathrm{mM} \mathrm{NaCl}, 10 \mathrm{mM}$ imidazole and $1 \mathrm{mg} / \mathrm{mL}$ lysozyme) and sonicated $8 \times 30 \mathrm{sec}(50 \%$ of the full power). After centrifugation (12000 rpm) for $25 \mathrm{~min}$ at $4^{\circ} \mathrm{C}$, the supernatant was applied onto nickel-charged agarose column (Qiagen). Histidine tagged proteins bound to nickel-affinity resins were eluted with $500 \mathrm{mM}$ imidazole. NmeGp1Sd protein was applied to a PD-10 desalting gel filtration column (Sephadex, GE Healthcare) equilibrated with $25 \mathrm{~mL}$ of $\mathrm{Nm} 23$ buffer [57] (20 mM HEPES at $\mathrm{pH} 7.9,5 \mathrm{mM} \mathrm{MgCl}_{2}, 0.1 \mathrm{mM}$ EDTA, $2 \mathrm{mM}$ DTT and $0.1 \mathrm{M} \mathrm{KCl}$ ) and then eluted with $3.5 \mathrm{~mL}$ of Nm23 buffer. The polyhistidine tag was released from the fusion protein by cleaving with thrombin (Sigma) (2 $\mathrm{U} / 40 \mu \mathrm{g}$ of fusion protein) for 16 hours at room temperature. The human Nme1 protein was a kind donation of Dr. Ioan Lascu (Institut de Biochemie et Genetique Cellulaires-IBGC, Bordeaux, France).

\section{Protein cross-linking with glutaraldehyde}

Oligomerization of the recombinant protein NmeGp1Sd was performed in PBS buffer as described by Kim et al. [38] for human Nme1 proteins with modified conditions: eight $\mu \mathrm{g}$ of sponge NmeGp1Sd was pre-incubated in PBS at room temperature and $25 \mathrm{mM}$ of glutaraldehyde was added to initiate the cross-linking. Following 5 min incubation, the reaction was quenched with 0.2 $\mathrm{M}$ Tris- $\mathrm{HCl}, \mathrm{pH} \mathrm{7.5}$, for $15 \mathrm{~min}$ at room temperature. The reaction product was subjected to $12.5 \%$ SDSPAGE and visualized by protein staining with Coomassie brilliant blue.

\section{Gel filtration chromatography}

Recombinant human Nme1 and sponge NmeGp1Sd were loaded onto Bio-Sil SEC 250 gel-filtration columns $(300 \mathrm{~mm} \times 7.8 \mathrm{~mm})$ and eluted with Nm23 buffer [57] at a flow rate of $0.5 \mathrm{~mL} / \mathrm{min}$ (BioLogic Duo Flow, BIORAD). Peak fractions were used for NDP kinase assay.

\section{DNA-binding assay}

The DNA-binding activity of the NmeGp1Sd protein was assayed in vitro as described [58]. Reactions contained $30 \mathrm{nM}$ of single-stranded circular DNA from bacteriophage $\varphi$ X174 (NEB). The amount of purified NmeGp1Sd protein is indicated (Figure 3). Human proteins Nme1 and 2 were used as controls. The reaction mixture was incubated in $20 \mu \mathrm{l}$ of buffer containing
$40 \mathrm{mM}$ Tris-acetate $(\mathrm{pH} 7.5)$ and $12 \mathrm{mM} \mathrm{MgCl} 2$. After 15 min of incubation at $37^{\circ} \mathrm{C}, 4 \mu \mathrm{L}$ of $40 \mathrm{mM}$ Tris-acetate $(\mathrm{pH} 7.5)$ containing $50 \%$ glycerol and $0.01 \%$ bromophenol blue was added and the products were immediately subjected to gel electrophoresis at $3 \mathrm{~V} / \mathrm{cm}$ in $0.6 \%$ agarose for 3 hours using $40 \mathrm{mM}$ Tris-acetate $(\mathrm{pH} 7.5)$ and $1 \mathrm{mM}$ EDTA as running buffer.

\section{NDP kinase assay}

Enzyme-coupled Reaction-NDPK activity was measured using a coupled pyruvate kinase-lactate dehydrogenase assay $[57,59]$. Five hundred $\mu \mathrm{L}$ of reaction mixtures were incubated in quartz cuvettes at room temperature in the presence of ATP as phosphate donor and dTDP as phosphate acceptor. The final concentrations were as follows: $50 \mathrm{mM}$ Tris- $\mathrm{HCl} \mathrm{pH} \mathrm{7.4,} 75 \mathrm{mM} \mathrm{KCl,} 5 \mathrm{mM}$ $\mathrm{MgCl}_{2}, 1 \mathrm{mM}$ phosphoenolpyruvate, $0.1 \mathrm{mg} / \mathrm{mL} \mathrm{NADH}$, $1 \mathrm{mM}$ ATP, $0.2 \mathrm{mM}$ dTDP, $2 \mathrm{U} / \mathrm{mL}$ of pyruvate kinase, $2.5 \mathrm{U} / \mathrm{mL}$ of lactate dehydrogenase, and $1 \mathrm{mg} / \mathrm{mL}$ bovine serum albumin. All reagents and enzymes were purchased from Sigma. Reactions were initiated by the addition of $50 \mathrm{ng}$ of NmeGp1Sd enzyme and activity was monitored in an Ultrospec ${ }^{\circledR} 2100$ pro (Amersham Pharmacia Biotech, USA) measuring the decrease in absorbance at $340 \mathrm{~nm}$. The control reactions omitting NmeGp1Sd produced minor rates but were subtracted from the NmeGp1Sd values. The same reaction was performed with human Nme1 enzyme.

\section{Plasmid cleavage assays}

We cloned the double stranded 57-bp NHE (nuclease hypersensitive element) of the $c$-myc promoter (5'GATCCCCAGTCTCCTCCCCACCTTCCCCACCCTC CCCACCCTCCCCATAAGCGAATT-3') insert into pUC19 plasmid. Recombinant plasmid pUC19-NHE (500 ng) was incubated with $500 \mathrm{ng}$ of NmeGp1Sd protein. The reaction was assembled in $50 \mathrm{mM} \mathrm{KCl}$, $10 \mathrm{mM} \mathrm{MgCl}_{2}$, $50 \mathrm{mM}$ Tris $\mathrm{HCl} \mathrm{pH}$ 7.5, $0.5 \mathrm{mM}$ DTT, $30 \mu \mathrm{g} / \mathrm{mL} \mathrm{BSA}$ and incubated $30 \mathrm{~min}$ at $20^{\circ} \mathrm{C}$. The reaction was terminated with $1 \%$ SDS, $10 \mathrm{mM}$ EDTA and proteinase $\mathrm{K}$ treatment $(200 \mu \mathrm{g} / \mathrm{mL})$ for $30 \mathrm{~min}$ at $55^{\circ} \mathrm{C}$. Topoisomerase I (Gibco BRL), Nme1 and Nme2 proteins were used as controls and were processed in the same conditions. Samples were analyzed in 1\% agarose gel in TAE buffer and than stained with $0.5 \mu \mathrm{g} / \mathrm{mL}$ ethidium bromide for $30 \mathrm{~min}$.

\section{Cell culture}

Human head and neck tumor cell line HEp-2 (squamous cell carcinoma of the larynx) was obtained from ATCC, while CAL 27 (squamous cell carcinoma of the tongue, ATCC) were a kind donation of Dr. Jeannine Gioanni, Centre Antoine Lacasagne, Nice, France. Both cell lines were cultured in Dulbecco's modified Eagle medium 
(DMEM, Invitrogen) supplemented with $10 \%$ fetal bovine serum (FBS, Invitrogen), $2 \mathrm{mM}$ glutamine, $100 \mathrm{U} / \mathrm{mL}$ penicillin and $100 \mu \mathrm{g} / \mathrm{mL}$ streptomycin in humidified chamber with $5 \% \mathrm{CO}_{2}$ at $37^{\circ} \mathrm{C}$.

\section{Transient transfections and laser scanning confocal microscopy}

Twenty four hours before transfection $3 \times 10^{4} \mathrm{HEp}-2$ cells were seeded on eight-well culture slides (BD-Falcon) to obtain $60 \%$ confluence. Each chamber was transfected with $1 \mu \mathrm{g}$ of plasmid DNA: pEGFPN1-Nme1, pEGFPN1-NmeGp1Sd, or cotransfected with pDsRedN1-NmeGp1Sd and pEGFPN1-Nme1 (0.5 $\mu \mathrm{g}$ of each construct) using Lipofectamine reagent (Invitrogen) according to the manufacturer's instructions. Forty eight hours post transfection the cells were washed with PBS (phosphate-buffered saline, $\mathrm{pH} 7.5$ ), fixed in $4 \%$ formaldehyde, and mounted in mounting medium (DAKO) supplemented with $1 \mu \mathrm{g} / \mathrm{mL}$ 4,6-diamino-2-phenylindole (DAPI) (Sigma) for nuclear staining.

Fluorescent images were obtained by Leica TCS SP2 AOBS laser scanning confocal microscopy equipped with HCX PL APO $\lambda$-Blue $63 \times 1.4$ objective. GFP was excited by $488 \mathrm{~nm}$ laser line, DsRed using $543 \mathrm{~nm}$, and DAPI using $405 \mathrm{~nm}$ laser line.

\section{Preparation of stably transfected CAL 27 clones}

Stably transfected cell lines were prepared as described previously [60]. In brief, CAL 27 cells were transfected using Lipofectamine reagent (Invitrogen) with pcDNA3FLAG/NmeGp1Sd and pcDNA3 as control. Post transfection the cells were resuspended and incubated in DMEM supplemented with geneticin (Sigma) until development of resistant colonies. Positive clones were screened by Western blotting, propagated and frozen until further usage. The clones with overexpressing FLAG/Nme1 (K1 and K2) have been formed in the exact same way and are the same ones used in [39].

\section{Immunoprecipitation and Western blotting}

For detection of Nme1/NmeGp1Sd complex formation $1 \times 10^{6}$ CAL 27 cells stably transfected with "empty" vector (K0), as well as CAL 27 cells stably expressing FLAG/NmeGp1Sd (S1 and S2) and FLAG/Nme1 expressing clones (K1 and $\mathrm{K} 2$ ) were seeded on 6-well plates overnight. Immunoprecipitation of FLAG/Nme1 was performed using anti-FLAG M2 affinity gel (Sigma) according to manufacturer's instructions.

The proteins were separated by SDS-PAGE and electrotransfered to an Imobilon-PSQ membrane (Milipore). The membranes were incubated with anti-Nme1 antibody (Beckton-Dickinson) or with anti-FLAG M2 antibody (Sigma) and Nme1 antibody for detection of complex formation. Protein bands were visualized using
Chemiluminiscence blotting substrate-POD (Roche) on Lumi-Film-Chemiluminiscent Detection Film (Roche).

\section{Migration in Boyden chambers}

Boyden chamber assay was done as described before [39]. Briefly, two six well plates were seeded with $3 \times$ $10^{5}$ cells (CAL 27, CAL 27 stably transfected with "empty" vector, CAL 27 constitutively overexpressing FLAG/Nme1 (K1 and K2) and CAL 27 constitutively expressing NmeGp1Sd (S1 and S2)) overnight. After attachment the cells were starved in serum free medium for 24 hours at $37^{\circ} \mathrm{C}$ in a humidified chamber. After starvation the cell suspension $\left(4 \times 10^{5}\right.$ cells $)$ was added to the upper chamber of Cell Culture Inserts (BecktonDickinson) and allowed to settle down for $10 \mathrm{~min}$. DMEM supplemented with 10\% FBS served as a chemoattractant and was added to the lower chamber. Cells were allowed to migrate for 20 hours. Nonmigratory cells were detached with cotton swabs, membranes were fixed in $4 \%$ formaldehyde, stained with $0.1 \%$ crystal violet, cut out from the inserts, mounted in mounting medium (DAKO) and analyzed by light microscopy. The migrated cells were analyzed at $400 \times$ magnitude. Three images per membrane were acquired and analyzed in ImageJ program http://rsbweb.nih.gov/ij/index.html which measured the area of the membrane covered with migrated cells.

\section{Additional material}

Additional file 1: Promoter regions. The structure of NmeGp 1 promoter regions from sponges $S$. domuncula and $A$. queenslandica. The most plausible putative binding sites for transcription factors identified by TFSEARCH are marked. Motifs shared with human Nmel promoter region are boxed. Arrows denote the orientation of motifs. TSS transcription start site.

Additional file 2: Maximum parsimony phylogenetic tree of Group I Nme members. Bootstrap values inferred from 1000 replicates are shown next to the branches (maximum parsimony/neighbour joining support). Accession numbers of sequences used are given in brackets after species names.

Additional file 3: Multiple sequence alignment of NmeGp1 proteins Human (Nme1 and Nme2), choanoflagellate Monosiga brevicollis (NmeGp1Mb) and sponges Suberites domuncula (NmeGp1Sd), Amphimedon queenslandica (NmeGp1Aq), Sycon raphanus (NmeGp1SrA and NmeGp1SrB) and Leucetta chagosensis (NmeGp1LcA and NmeGp1LcB) proteins were aligned. Enzyme active site amino acids and amino acids necessary for the association of subunits on the hexamer are conserved and marked with *.

\section{Acknowledgements}

This work was funded by Croatian MSES grants 098-0982913-2478 (H. Ćetković) and 098-09824642513 (M. Herak Bosnar). Prof. Dr. WEG Müller is gratefully acknowledged for S. domuncula cDNA and genomic library. We are grateful to Dr. Robert Belužić for gel filtration chromatography analysis and Dr. Mary Sopta for proofreading the manuscript. We are thankful to the three anonymous reviewers for their constructive comments which greatly improved the quality of the manuscript. 


\section{Author details}

'Division of Molecular Biology, Ruđer Bošković Institute, Bijenička cesta 54, 10002 Zagreb, Croatia. ²Division of Molecular Medicine, Ruđer Bošković Institute, Bijenička cesta 54, 10002 Zagreb, Croatia.

\section{Authors' contributions}

DP carried out the molecular genetic studies, biochemical assays and the sequence analysis. MHB and RB carried out colocalization, immunoprecipitation and migration experiments. MD carried out stable clone preparation. AM participated in the biochemical assays and $\mathrm{MH}$ performed phylogenetic analysis. HĆ conceived and oversaw the project, participated in the sequence analysis and molecular genetic studies. $H C_{\text {, }}$ $\mathrm{MHB}, \mathrm{DP}$ and $\mathrm{MH}$ wrote the manuscript. All authors read and approved the final manuscript.

Received: 11 August 2010 Accepted: 1 April 2011 Published: 1 April 2011

\section{References}

1. Bilitou A, Watson J, Gartner A, Ohnuma S: The NM23 family in development. Mol Cell Biochem 2009, 329(1-2):17-33.

2. Desvignes $T$, Pontarotti $P$, Fauvel $C$, Bobe J: Nme protein family evolutionary history, a vertebrate perspective. BMC Evol Biol 2009, 9:256.

3. Steeg PS, Bevilacqua G, Kopper L, Thorgeirsson UP, Talmadge JE, Liotta LA Sobel ME: Evidence for a novel gene associated with low tumor metastatic potential. J Natl Cancer Inst 1988, 80(3):200-204.

4. Rinker-Schaeffer CW, O'Keefe JP, Welch DR, Theodorescu D: Metastasis suppressor proteins: discovery, molecular mechanisms, and clinical application. Clin Cancer Res 2006, 12(13):3882-3889.

5. Hartsough MT, Steeg PS: Nm23/nucleoside diphosphate kinase in human cancers. J Bioenerg Biomembr 2000, 32(3):301-308.

6. Hennessy C, Henry JA, May FE, Westley BR, Angus B, Lennard TW: Expression of the antimetastatic gene $\mathrm{nm} 23$ in human breast cancer: an association with good prognosis. J Natl Cancer Inst 1991, 83(4):281-285.

7. Ilijas M, Pavelic K, Sarcevic B, Kapitanovic S, Kurjak A, Stambrook PJ, Gluckman JL, Pavelic ZP: Expression Of Nm23-H1 Gene In Squamous Cell Carcinoma Of The Cervix Correlates With 5-Year Survival. International Journal of Oncology 1994, 5(6):1455-1457.

8. Boissan M, Lacombe ML: Nm23/NDP kinases in hepatocellular carcinoma. J Bioenerg Biomembr 2006, 38(3-4):169-175.

9. Gilles AM, Presecan E, Vonica A, Lascu I: Nucleoside diphosphate kinase from human erythrocytes. Structural characterization of the two polypeptide chains responsible for heterogeneity of the hexameric enzyme. J Biol Chem 1991, 266(14):8784-8789.

10. MacDonald NJ, De la Rosa A, Benedict MA, Freije JM, Krutsch H, Steeg PS: A serine phosphorylation of $\mathrm{Nm} 23$, and not its nucleoside diphosphate kinase activity, correlates with suppression of tumor metastatic potential. J Biol Chem 1993, 268(34):25780-25789.

11. Wagner PD, Vu ND: Phosphorylation of ATP-citrate lyase by nucleoside diphosphate kinase. J Biol Chem 1995, 270(37):21758-21764.

12. Wagner PD, Vu ND: Histidine to aspartate phosphotransferase activity of $\mathrm{nm} 23$ proteins: phosphorylation of aldolase C on Asp-319. Biochem J 2000, 346(Pt 3):623-630.

13. Ma D, McCorkle JR, Kaetzel DM: The metastasis suppressor NM23-H1 possesses 3'-5' exonuclease activity. J Biol Chem 2004, 279(17):18073-18084.

14. Postel EH: Multiple biochemical activities of NM23/NDP kinase in gene regulation. J Bioenerg Biomembr 2003, 35(1):31-40.

15. Lombardi $D$, Lacombe ML, Paggi MG: nm23: unraveling its biological function in cell differentiation. J Cell Physiol 2000, 182(2):144-149.

16. Lombardi D, Palescandolo E, Giordano A, Paggi MG: Interplay between the antimetastatic $\mathrm{nm} 23$ and the retinoblastoma-related $\mathrm{Rb} 2 / \mathrm{p} 130$ genes in promoting neuronal differentiation of PC12 cells. Cell Death Differ 2001, 8(5):470-476.

17. Gervasi F, D’Agnano I, Vossio S, Zupi G, Sacchi A, Lombardi D: nm23 influences proliferation and differentiation of PC12 cells in response to nerve growth factor. Cell Growth Differ 1996, 7(12):1689-1695.

18. Lakso M, Steeg PS, Westphal H: Embryonic expression of nm23 during mouse organogenesis. Cell Growth Differ 1992, 3(12):873-879.
19. Amrein L, Barraud P, Daniel JY, Perel Y, Landry M: Expression patterns of nm23 genes during mouse organogenesis. Cell Tissue Res 2005, 322(3):365-378.

20. Kang $Y$, Lee DC, Han J, Yoon S, Won M, Yeom JH, Seong MJ, Ko JJ, Lee KA, Lee $\mathrm{K}$, et al: NM23-H2 involves in negative regulation of Diva and $\mathrm{Bcl} 2 \mathrm{~L} 10$ in apoptosis signaling. Biochem Biophys Res Commun 2007, 359(1):76-82.

21. Fan Z, Beresford PJ, Oh DY, Zhang D, Lieberman J: Tumor suppressor NM23-H1 is a granzyme A-activated DNase during CTL-mediated apoptosis, and the nucleosome assembly protein SET is its inhibitor. Cell 2003, 112(5):659-672.

22. Fournier HN, Albiges-Rizo C, Block MR: New insights into Nm23 control of cell adhesion and migration. J Bioenerg Biomembr 2003, 35(1):81-87.

23. Palacios F, Schweitzer JK, Boshans RL, D'Souza-Schorey C: ARF6-GTP recruits $\mathrm{Nm} 23-\mathrm{H} 1$ to facilitate dynamin-mediated endocytosis during adherens junctions disassembly. Nat Cell Biol 2002, 4(12):929-936.

24. Desvignes T, Pontarotti P, Bobe J: Correction: Nme Gene Family Evolutionary History Reveals Pre-Metazoan Origins and High Conservation between Humans and the Sea Anemone, Nematostella vectensis. PLoS One 2010, 5(11).

25. Pick KS, Philippe $H$, Schreiber F, Erpenbeck D, Jackson DJ, Wrede $P$, Wiens M, Alie A, Morgenstern B, Manuel M, et al: Improved phylogenomic taxon sampling noticeably affects nonbilaterian relationships. Mol Biol Evol 2010, 27(9):1983-1987

26. Philippe H, Derelle R, Lopez P, Pick K, Borchiellini C, Boury-Esnault N, Vacelet J, Renard E, Houliston E, Quéinnec E, et al: Phylogenomics Revives Traditional Views on Deep Animal Relationships. Current biology: CB 2009, 19(8):706-712.

27. Dunn CW, Hejnol A, Matus DQ, Pang K, Browne WE, Smith SA, Seaver E, Rouse GW, Obst M, Edgecombe GD, et al: Broad phylogenomic sampling improves resolution of the animal tree of life. Nature 2008, 452(7188):745-749.

28. Hejnol A, Obst M, Stamatakis A, Ott M, Rouse GW, Edgecombe GD, Martinez P, Baguna J, Bailly X, Jondelius U, et al: Assessing the root of bilaterian animals with scalable phylogenomic methods. Proc Biol Sci 2009, 276(1677):4261-4270.

29. Cetkovic H, Mikoc A, Muller WE, Gamulin V: Ras-like small GTPases form a large family of proteins in the marine sponge Suberites domuncula. J Mol Evol 2007, 64(3):332-341.

30. Perina D, Cetkovic H, Harcet M, Premzl M, Lukic-Bilela L, Muller WE, Gamulin V: The complete set of ribosomal proteins from the marine sponge Suberites domuncula. Gene 2006, 366(2):275-284.

31. Cetkovic H, Muller WE, Gamulin V: Bruton tyrosine kinase-like protein, BtkSD, is present in the marine sponge Suberites domuncula. Genomics 2004, 83(4):743-745

32. Srivastava M, Simakov O, Chapman J, Fahey B, Gauthier ME, Mitros T, Richards GS, Conaco C, Dacre M, Hellsten U, et al: The Amphimedon queenslandica genome and the evolution of animal complexity. Nature 2010, 466(7307):720-726.

33. Harcet M, Roller M, Cetkovic H, Perina D, Wiens M, Muller WE, Vlahovicek K: Demosponge EST sequencing reveals a complex genetic toolkit of the simplest metazoans. Mol Biol Evol 2010, 27(12):2747-2756.

34. Harcet M, Lukic-Bilela L, Cetkovic H, Muller WEG, Gamulin V: Identification and analysis of CDNAs encoding two nucleoside diphosphate kinases (NDPK/Nm23) from the marine sponge Suberites domuncula. Croat Chem Acta 2005, 78(3):343-348.

35. de la Rosa A, Mikhak B, Steeg PS: Identification and characterization of the promoter for the human metastasis suppressor gene $\mathrm{nm} 23-\mathrm{H} 1$. Arch Med Res 1996, 27(3):395-401.

36. Chen $\mathrm{HC}$, Wang L, Banerjee S: Isolation and characterization of the promoter region of human $\mathrm{nm} 23-\mathrm{H} 1$, a metastasis suppressor gene. Oncogene 1994, 9(10):2905-2912.

37. Ouatas T, Abdallah B, Gasmi L, Bourdais J, Postel E, Mazabraud A: Three different genes encode NM23/nucleoside diphosphate kinases in Xenopus laevis. Gene 1997, 194(2):215-225.

38. Kim Yl, Park S, Jeoung DI, Lee H: Point mutations affecting the oligomeric structure of Nm23-H1 abrogates its inhibitory activity on colonization and invasion of prostate cancer cells. Biochem Biophys Res Commun 2003, 307(2):281-289. 
39. Bago R, Pavelic J, Vlahovicek GM, Bosnar MH: Nm23-H1 Promotes Adhesion of CAL 27 Cells In Vitro. Molecular Carcinogenesis 2009, 48(9):779-789.

40. Postel EH: Cleavage of DNA by human NM23-H2/nucleoside diphosphate kinase involves formation of a covalent protein-DNA complex. J Biol Chem 1999, 274(32):22821-22829.

41. Bosnar MH, De Gunzburg J, Bago R, Brecevic L, Weber I, Pavelic J: Subcellular localization of A and B Nm23/NDPK subunits. Exp Cell Res 2004, 298(1):275-284

42. Muller WE, Bohm M, Grebenjuk VA, Skorokhod A, Muller IM, Gamulin V: Conservation of the positions of metazoan introns from sponges to humans. Gene 2002, 295(2):299-309.

43. Cetkovic H, Grebenjuk VA, Muller WE, Gamulin V: Src proteins/src genes: from sponges to mammals. Gene 2004, 342(2):251-261.

44. Annesley SJ, Fisher PR: Dictyostelium discoideum-a model for many reasons. Mol Cell Biochem 2009, 329(1-2):73-91.

45. Nallamothu G, Dammai V, Hsu T: Developmental function of Nm23/awd: a mediator of endocytosis. Mol Cell Biochem 2009, 329(1-2):35-44.

46. Domazet-Loso T, Tautz D: Phylostratigraphic tracking of cancer genes suggests a link to the emergence of multicellularity in metazoa. BMC Biol 2010, 8:66.

47. Muller WEG: Review: How was metazoan threshold crossed? The hypothetical Urmetazoa. Comp Biochem Physiol A-Mol Integr Physiol 2001 129(2-3):433-460.

48. Newman SA, Bhat R: Dynamical patterning modules: a "pattern language" for development and evolution of multicellular form. Int J Dev Biol 2009, 53(5-6):693-705.

49. Fahey B, Degnan BM: Origin of animal epithelia: insights from the sponge genome. Evol Dev 2010, 12(6):601-617.

50. Degnan SM, Degnan BM: The initiation of metamorphosis as an ancient polyphenic trait and its role in metazoan life-cycle evolution. Philos Trans R Soc Lond B Biol Sci 2010, 365(1540):641-651.

51. Muller WEG: The origin of metazoan complexity: Porifera as integrated animals. Integr Comp Biol 2003, 43(1):3-10.

52. Seack J, Perovic S, Gamulin V, Schroder HC, Beutelmann P, Muller IM, Muller WE: Identification of highly conserved genes: SNZ and SNO in the marine sponge Suberites domuncula: their gene structure and promoter activity in mammalian cells(1). Biochim Biophys Acta 2001, 1520(1):21-34.

53. Thompson JD, Higgins DG, Gibson TJ: CLUSTAL W: improving the sensitivity of progressive multiple sequence alignment through sequence weighting, position-specific gap penalties and weight matrix choice. Nucleic Acids Res 1994, 22(22):4673-4680.

54. Tamura K, Dudley J, Nei M, Kumar S: MEGA4: Molecular Evolutionary Genetics Analysis (MEGA) software version 4.0. Mol Biol Evol 2007, 24(8):1596-1599.

55. Milanesi L, Muselli M, Arrigo P: Hamming-Clustering method for signals prediction in $5^{\prime}$ and $3^{\prime}$ regions of eukaryotic genes. Comput Appl Biosci 1996, 12(5):399-404.

56. Heinemeyer $\mathrm{T}$, Wingender $\mathrm{E}$, Reuter I, Hermjakob H, Kel AE, Kel OV, Ignatieva EV, Ananko EA, Podkolodnaya OA, Kolpakov FA, et al: Databases on transcriptional regulation: TRANSFAC, TRRD and COMPEL. Nucleic Acids Res 1998, 26(1):362-367.

57. Postel EH, Ferrone CA: Nucleoside diphosphate kinase enzyme activity of $\mathrm{NM} 23-\mathrm{H} 2 / \mathrm{PuF}$ is not required for its DNA binding and in vitro transcriptional functions. J Biol Chem 1994, 269(12):8627-8630.

58. Galvao CW, Pedrosa FO, Souza EM, Yates MG, Chubatsu LS, Steffens MB: Expression, purification, and DNA-binding activity of the Herbaspirillum seropedicae RecX protein. Protein Expr Purif 2004, 35(2):298-303.

59. Agarwal RP, Robison B, Parks RE Jr: Nucleoside diphosphokinase from human erythrocytes. Methods Enzymol 1978, 51:376-386.

60. Bosnar MH, Dubravcic K, Bago R, Pavelic J: Head and neck tumor cells exhibit altered proliferation upon overexpression of $n m 23$ genes. Croat Chem Acta 2008, 81(1):183-189.

doi:10.1186/1471-2148-11-87

Cite this article as: Perina et al:: Sponge non-metastatic Group I Nme gene/protein - structure and function is conserved from sponges to humans. BMC Evolutionary Biology 2011 11:87.

\section{Submit your next manuscript to BioMed Central and take full advantage of:}

- Convenient online submission

- Thorough peer review

- No space constraints or color figure charges

- Immediate publication on acceptance

- Inclusion in PubMed, CAS, Scopus and Google Scholar

- Research which is freely available for redistribution 